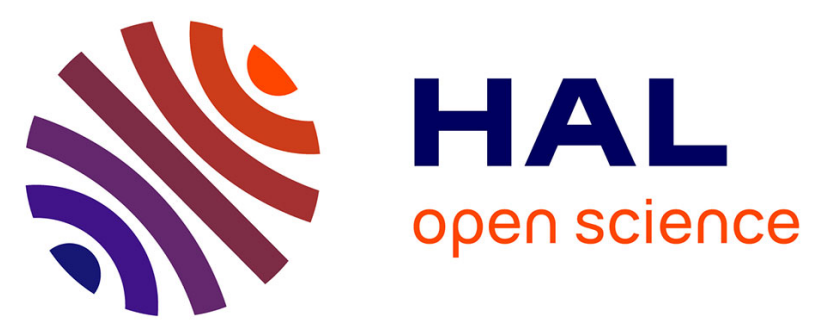

\title{
Electron temperature anisotropy associated to field-aligned currents in the Earth's magnetosphere inferred from Rosetta MIP-RPC observations during 2009 flyby
}

Christian Béghin, Michel Hamelin, Jean-Pierre Lebreton, Xavier Vallieres, Jérôme Moré, Pierre Henri

\section{To cite this version:}

Christian Béghin, Michel Hamelin, Jean-Pierre Lebreton, Xavier Vallieres, Jérôme Moré, et al.. Electron temperature anisotropy associated to field-aligned currents in the Earth's magnetosphere inferred from Rosetta MIP-RPC observations during 2009 flyby. Journal of Geophysical Research Space Physics, 2017, 122 (7), pp.6964-6977. 10.1002/2017JA024096 . insu-01544697

\section{HAL Id: insu-01544697 https://hal-insu.archives-ouvertes.fr/insu-01544697}

Submitted on 16 Nov 2017

HAL is a multi-disciplinary open access archive for the deposit and dissemination of scientific research documents, whether they are published or not. The documents may come from teaching and research institutions in France or abroad, or from public or private research centers.
L'archive ouverte pluridisciplinaire HAL, est destinée au dépôt et à la diffusion de documents scientifiques de niveau recherche, publiés ou non, émanant des établissements d'enseignement et de recherche français ou étrangers, des laboratoires publics ou privés. 


\section{Journal of Geophysical Research: Space Physics}

\section{RESEARCH ARTICLE}

10.1002/2017JA024096

\section{Special Section: \\ Observations, Simulations, and Theory of Electric Currents in the Solar System}

Key Points:

- The prime significant point of this work is the first attempt to derive the perpendicular electron temperature component versus the magnetic field by using the Bernstein's Fq's modes properties

- The two active-passive MIP modes allowed subsequently to determine both electron temperature components $T_{e \perp}$ and $T_{e / /}$, and their anisotropy ratio within sets of magnetospheric FACs tubes

- Complementary data returns, such as determination of the Beta ratio electron temperature/magnetic pressure, bring new inputs to our understanding of the FACs tubes dynamic confinement

Supporting Information:

- Supporting Information S1

Correspondence to:

C. Béghin,

christian.beghin@cnrs-orleans.fr

Citation:

Béghin, C., M. Hamelin, J. P. Lebreton, X. Vallieres, J. Moré, and P. Henri (2017), Electron temperature anisotropy associated to field-aligned currents in the Earth's magnetosphere inferred from Rosetta MIP-RPC observations during 2009 flyby, J. Geophys. Res. Space Physics, 122, 6964-6977, doi:10.1002/ 2017JA024096.

Received 28 FEB 2017

Accepted 16 JUN 2017

Accepted article online 20 JUN 2017

Published online 17 JUL 2017

o2017. American Geophysical Union. All Rights Reserved.
Electron temperature anisotropy associated to field-aligned currents in the Earth's magnetosphere inferred from Rosetta MIP-RPC observations during 2009 flyby

\author{
C. Béghin ${ }^{1}$ iD, M. Hamelin ${ }^{2}$, J. P. Lebreton ${ }^{1}$, X. Vallieres ${ }^{1}$, J. Moré ${ }^{1}$, and P. Henri ${ }^{1}$ iD \\ ${ }^{1}$ LPC2E-CNRS-Université d'Orléans, Orléans, France, ${ }^{2}$ Sorbonne Universités, UPMC Univ. Paris 06, LATMOS-IPSL, UMR 8190, \\ Paris, France
}

Abstract A new approach is proposed for data interpretation of the Mutual Impedance Probe (MIP) instrument from the Rosetta Plasma Consortium (RPC) during the 2009 Earth's flyby gravity assist through the magnetosphere, from dusk to dawn regions. The spacecraft trajectory of $\pm 8 R_{E}$ (Earth's radius) was crossing several structures of field-aligned currents (FACs) and radiations belts on both legs of the closest approach (CA, $2.450 \mathrm{~km}$ altitude). As routinely revealed by several pioneering space missions, natural and forced electrostatic wave emissions called Fqs were observed over $\pm 3 R_{E}$ at around CA using a dedicated mode of the MIP instrument. These emissions are lying between consecutive harmonics of the electroncyclotron frequency, and their wavelength is perpendicular to the magnetic field lines. Provided that the Fq's wavelengths projected along the MIP antenna might be estimated, it is shown that the local value of the Larmor radius can be deduced; hence, the electron temperature component perpendicular to the magnetic field is subsequently derived. On the other hand, during the time of Fq's observations, the presence of VLF hiss emissions usually observed in these regions gives us the possibility to determine the electron temperature anisotropy associated to the electrostatic electron anisotropy instability according to the theoretical model proposed by Gary and Cairns (JGR, vol. 104, 1999). Significant dynamic constraints revealed by crossing successive series of FACs tubes are shown being controlled by this anisotropy, and the fact that the magnetic pressure is significantly larger than the thermal pressure suggests that the FACs lobes are nonforce free.

Plain Language Summary The prime significant point of this work is the first attempt to derive the perpendicular electron temperature component versus the magnetic field by using the Bernstein's Fq's modes properties. Complementary data returns, such as determination of the Beta ratio electron temperature/magnetic pressure, bring new inputs to our understanding of the FACs tubes dynamic confinement. The new approach proposed in this work for the treatment of Fq's resonances appears promising to be implemented for further space missions in magnetized plasmas of the planets in the solar system, in order to determinate the electron temperature perpendicular component, to reveal the possible existence of an electron temperature anisotropy and thus the force ratio between the two components of electron and magnetic thermal pressures.

\section{Introduction}

The principle of mutual impedance probes (MIP), proposed by Storey et al. [1969] for the diagnostic of natural plasmas, such as the Earth's ionosphere-magnetosphere, was implemented for the first time in the frequency range of the lower hybrid resonance (LHR), both at middle and equatorial latitudes on board of sounding rockets by Béghin [1971] and Béghin and Debrie [1972]. Soon after, the domain of the electron plasma frequency $\left(F_{\mathrm{p}}\right)$ was the objective of further rocket flights in both equatorial and polar regions [Chasseriaux et al., 1972; Béghin et al., 1976] and for the study of the oblique resonance cone [Michel et al., 1975]. An improved MIP device for lower density and higher electron temperature was then flown on board the GEOS satellites [Décréau et al., 1978], followed by the Arcad-3 satellite [Béghin et al., 1982] and by other space missions projects, including Rosetta.

While most of these previous missions were devoted to specific studies of different regions in the Earth's magnetosphere, such as the plasmapause (GEOS) and polar-auroral regions (Arcad-3), the highest priority of the Rosetta Earth's swing-by was rather an Earth's gravity assist maneuver imposed by the major 

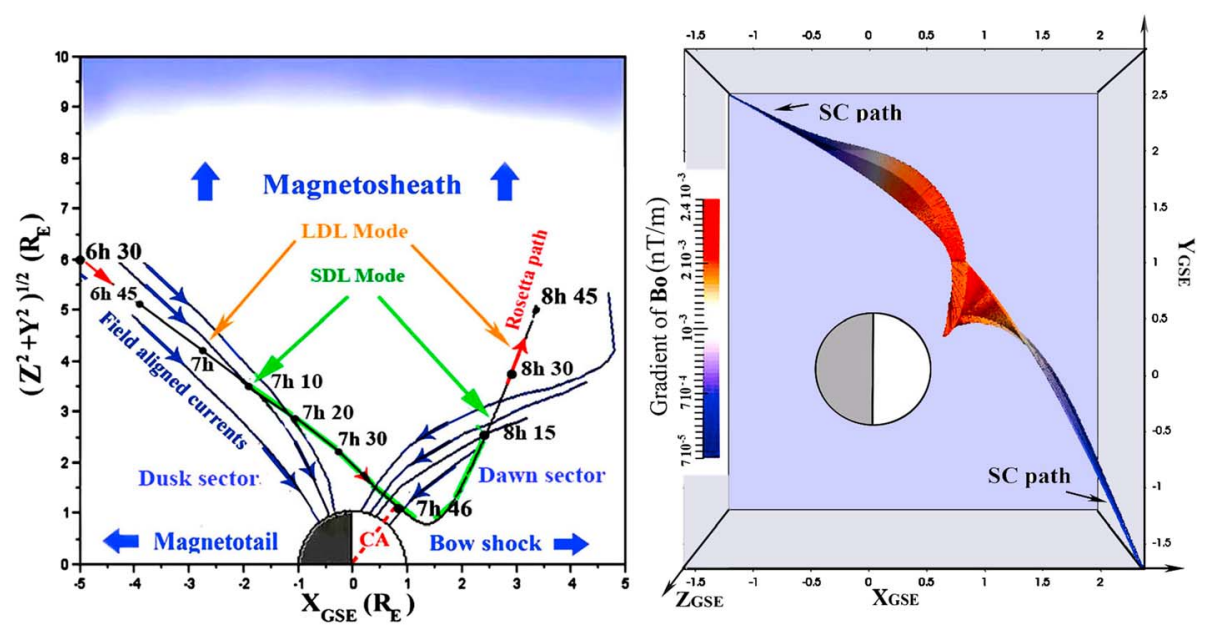

Figure 1. (left) Rosetta path in GSM coordinates plotted along a schematic field-aligned currents pattern according to Dungey's reconnection model [Dungey, 1961]. (right) Path of SDL mode section, with $\nabla B_{0}$ variations derived from onboard magnetometer plotted in 3-D along the geocentric coordinates (GSE).

objective of the mission toward the encounter with comet 67P Churyumov-Gerasimenko [Glassmeier et al., 2007a]. Nevertheless, the opportunity to switch on some of the RPC instruments [Carr et al., 2007] offered a valuable occasion for a significant test of truth for the MIP capabilities. As for the two first Earth's flybys on 2005 and 2007 [Trotignon et al., 2007b], the Rosetta trajectory on 13 November 2009 covered a large domain of $10 R_{E}$ (Earth radii) from dusk to dawn sectors on either side of the closest approach (CA $~ 2500 \mathrm{~km}$ ). The entire Rosetta trajectory in GSM coordinates from $6 \mathrm{~h} 30 \mathrm{~min}$ to $8 \mathrm{~h} 45$ UT (Figure 1, left) is plotted along a schematic model [Dungey, 1961] of magnetic reconnection of the Earth's magnetosphere through sets of currents connecting the solar wind bow shock to the magnetotail.

Similarly to what was anticipated for the upcoming encounter with the comet, the design of the MIP instrumentation offered different modes of operations [Trotignon et al., 2007a, 2007b]. For each MIP sequence, by anticipating the likely range of plasma characteristic parameters such as the Debye length $\left(\lambda_{D}\right)$ and the Larmor radius $(\rho)$, the best suited operation modes of preprogrammed sequences were uplinked sometime in advance from the Rosetta Mission Operations Centre (ESA-ESOC, Darmstadt, Germany). For the 2009 swing by, two operational modes were successively activated (Figure 1, left), named, respectively, LDL and SDL [Geiswiller et al., 2001a.], i.e., large Debye length and short Debye length in order to adapt the best MIP configuration to the anticipated plasma parameters.

The portion of Rosetta trajectory involving the SDL mode that is relevant to this work lies between $7 \mathrm{~h} 10$ and $8 \mathrm{~h} 15$ UT. Although the magnetospheric conditions at this date were pretty quiet, with $K_{p}<1$ (NOAA Base), the Rosetta trajectory revealed successive crossings of field aligned currents (Figure 1, left) that is the topic being developed in section 4. The first evidence of these structures appeared from the gradient variations of the magnetic field vector, as seen in the frame of Rosetta path (Figure 1, right) and defined as

$$
\widetilde{N} B o=\frac{\mathrm{d} B x}{\mathrm{~d} x} X+\frac{\mathrm{d} B y}{\mathrm{~d} y} Y+\frac{\mathrm{d} B z}{\mathrm{~d} z} Z
$$

The field-aligned currents are quasi-permanently observed in this region since long ago, even during quiet and moderately disturbed conditions [e.g., Lijima and Potemra, 1976; Mozer et al., 1979]. Most of these structures are known to be generated by high-energy particles of the solar wind that are diffracted and reflected back respectively by the bow shock and the magnetotail, before converging toward the auroral and eventually middle latitude regions (see reviews by Blanc et al. [2005] and Milan and Thorne [2007]).

The design of the SDL mode, involving the determination of Debye length and Larmor radius of maximum metric magnitude order, was anticipated to be relevant with calm condition models of midmagnetic latitudes along the $\pm 2 R_{E}$ portion of the Rosetta trajectory. Such estimate was subsequently validated by the actual profiles of the electron density and the associated range covered by the Debye length and the Larmor radius 


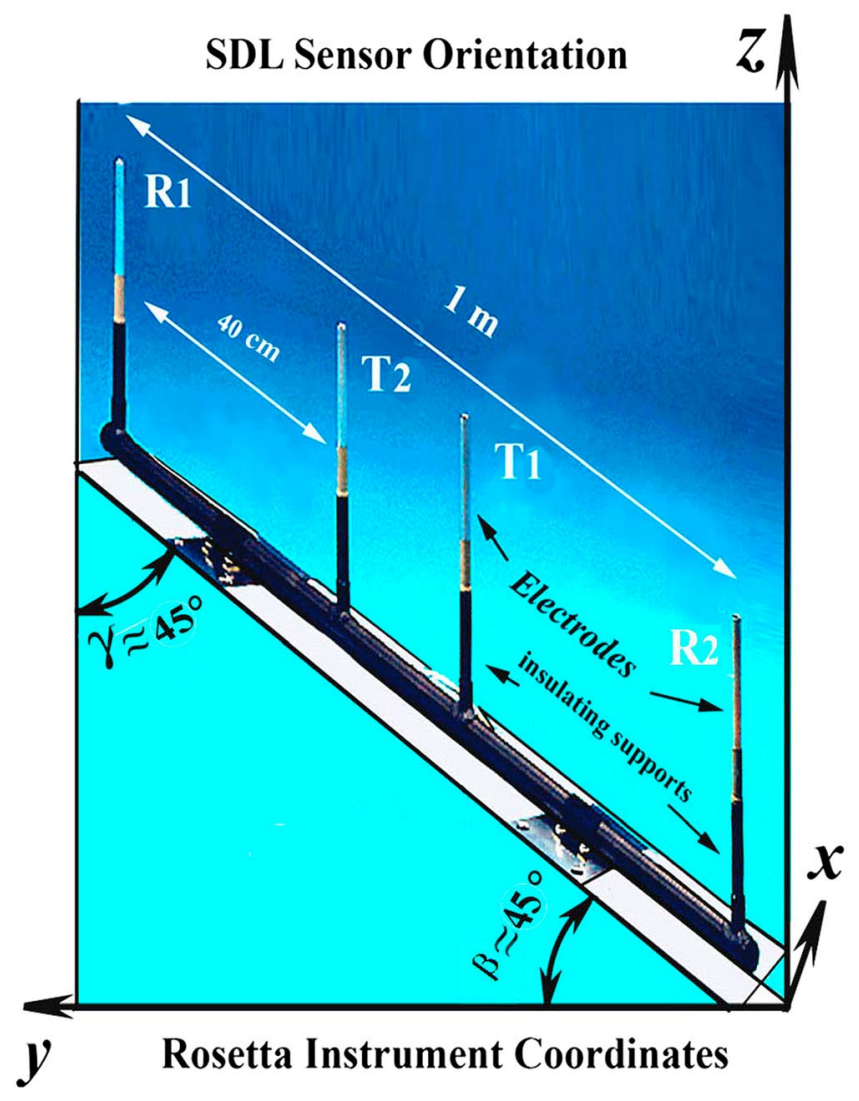

Figure 2. Configuration of the Rosetta SDL probe in spacecraft instrumentation coordinates. (section 2) from 2 to $20 \mathrm{~cm}$ and $20 \mathrm{~cm}$ to $2 \mathrm{~m}$, respectively. Contrary to conventional processing methods in weakly or unmagnetized plasmas [e.g., Chasseriaux et al., 1972], the electron temperature components are derived here (section 3 ) from a new method making use of properties of Bernstein's Fq waves that are propagating in the perpendicular direction to the steady magnetic field lines [e.g., Christiansen et al., 1978; Perraut et al., 1990].

\section{The MIP-SDL Mode Capability for Natural and Triggered Plasma Waves}

The basic concept of MIP sensors is making use of a transmission dipole driving a radio frequency (RF) of small amplitude and constant current, being associated to a highimpedance reception dipole, either parallel or perpendicular to each other [Storey et al., 1969]. Here the design of the SDL mode antenna [Geiswiller et al., 2001a; Trotignon et al., 2007a] is derived from the Arcad-3 mission [Béghin et al., 1982], using a probe antenna $1 \mathrm{~m}$ long (Figure 2), with the geometric configuration of dual aligned dipoles The nominal design of such antenna implies two transmission electrodes (T1-T2), both driven by an antiphase constant amplitude current lying within 28 to $1582 \mathrm{kHz}$ frequency range for the active mode. A double-probe antenna (R1-R2) is used for the reception signal, in either active or passive (natural waves) mode. For this nominal layout, different arrangements of the electrodes were tested during the calbration sequences. However, the selected best configuration for the Earth flyby was rather the "monopole emission" mode, that means a constant current driven only by the electrode T2, while the coherent return current comes from the induced charge distribution induced on the nearby conductive surfaces of the spacecraft ground floating voltage. The orientation of the SDL antenna in the Rosetta structure coordinates is shown in Figure 2, as an important data accounting for the determination of Fq's wavelengths perpendicular to the local magnetic field (see section 3.2).

\subsection{Determination of $\boldsymbol{F}_{\mathrm{p}}$ and $\boldsymbol{N}_{\mathrm{e}}$}

From the first MIP instruments implemented on board space missions in the Earth magnetosphere [Décréau et al., 1978; Béghin et al., 1982], the main resonance to be readily identified was the Upper Hybrid $\left(F_{\text {uh }}\right)$ defined as

$$
F_{\mathrm{uh}}=\left[\left(F_{\mathrm{p}}\right)^{2}+\left(F_{\mathrm{ce}}\right)^{2}\right]^{1 / 2}
$$

where $F_{\mathrm{ce}}$ and $F_{\mathrm{p}}$ are the electron cyclotron and plasma frequencies defined respectively as

$$
F_{\mathrm{ce}}=\frac{1}{2 \pi}\left(\frac{e B_{0}}{m_{\mathrm{e}}}\right) ; F_{\mathrm{p}}=\frac{1}{2 \pi}\left[\frac{N_{\mathrm{e}} e^{2}}{m_{\mathrm{e}} \varepsilon_{0}}\right]^{1 / 2}
$$

where $\left(m_{\mathrm{e}}, e, N_{\mathrm{e}}\right)$ are the electron mass, electric charge, and number density, respectively, $\varepsilon_{0}$ and $B_{0}$ are the 

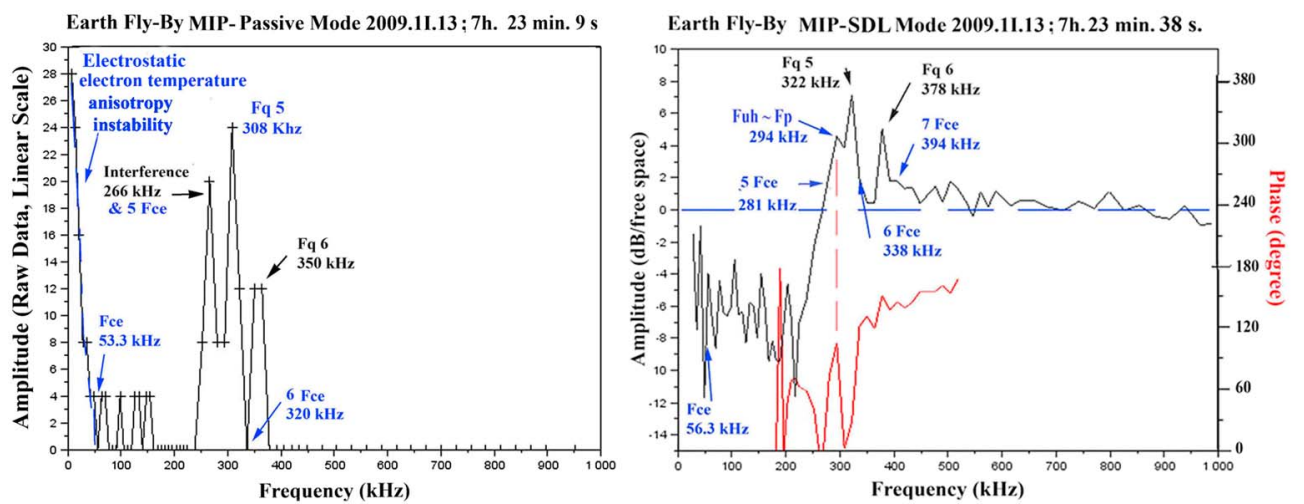

Figure 3. Example of SDL (left) passive and (right) active consecutive modes when $F_{\mathrm{p}} / F_{\mathrm{ce}} \gg 1$.

free-space permittivity and magnetic field amplitudes (all in SI units). For practical application we take: $F_{\mathrm{p}}=9$ $\left(N_{\mathrm{e}}\right)^{1 / 2}$ and $F_{\mathrm{ce}} \approx 2.8 \times 10^{-2} B_{0}$, with $N_{\mathrm{e}}(\mathrm{e} / \mathrm{cc}), B_{0}(\mathrm{nT})$, and frequencies in $\mathrm{kHz}$.

As long as the $F_{\mathrm{uh}}$ frequency might be identified, both values of $F_{\mathrm{p}}$ and $N_{\mathrm{e}}$ are unambiguously determined since the value of $F_{\text {ce }}$ is known thanks to the onboard measurements of local magnetic field performed by the Rosetta RPC fluxgate magnetometer MAG [Glassmeier et al., 2007b]. However, the identification of $F_{\mathrm{uh}}$ with respect to $F_{\mathrm{p}}$ in the MIP response is not always obvious, because the more the ratio $F_{\mathrm{p}} / F_{\text {ce }}$ is larger than 1 , the more the actual resonance becomes difficult to be differentiated from each other, as they are seen merging together in Figure 3.

Nevertheless, in such conditions the full MIP spectral response (amplitude and phase) allows us to determine the most likely value of the strongest resonance between $F_{\mathrm{p}}$ or $F_{\mathrm{uh}}$. Using the approximation of a collisionless weakly magnetized cold plasma $\left(F_{\mathrm{p}}>F_{\mathrm{ce}}\right)$, the behavior of diagonal elements of the dielectric tensor [Stix, 1962] led Storey et al. [1969] to predicting a 180-degree phase change (from real to imaginary part) of the MIP response through the plasma frequency singularity. Shortly thereafter, a phase shift of 90 to 180 degrees in the vicinity of $F_{\mathrm{p}}$ was indeed identified with MIP instruments in the magnetosphere [e.g., Décréau et al., 1978] and this interesting property was implemented in the design of high-time resolution self-oscillating MIP probes automatically locked on $F_{\mathrm{p}} \sim F_{\mathrm{uh}}$ frequencies [Béghin et al., 1982]. The above phase behavior at around $F_{\mathrm{p}}$ had been subsequently confirmed by numerical simulations of the Rosetta MIP instruments responses in a Maxwellian plasma [Geiswiller et al., 2001b]. The example of both active and passive modes SDL responses of the 2009 swing-by plotted in Figure 3 is lying in such a situation when $F_{\mathrm{p}}$ and $F_{\text {uh }}$ are merging. The $N_{\mathrm{e}}, F_{\mathrm{p}}$ and $F_{\mathrm{p}} / F_{\text {ce }}$ profiles variations, as derived according to the above procedure for the entire SDL sequence, are shown in Figure 4.

Parameters observed or inferred from Rosetta observations
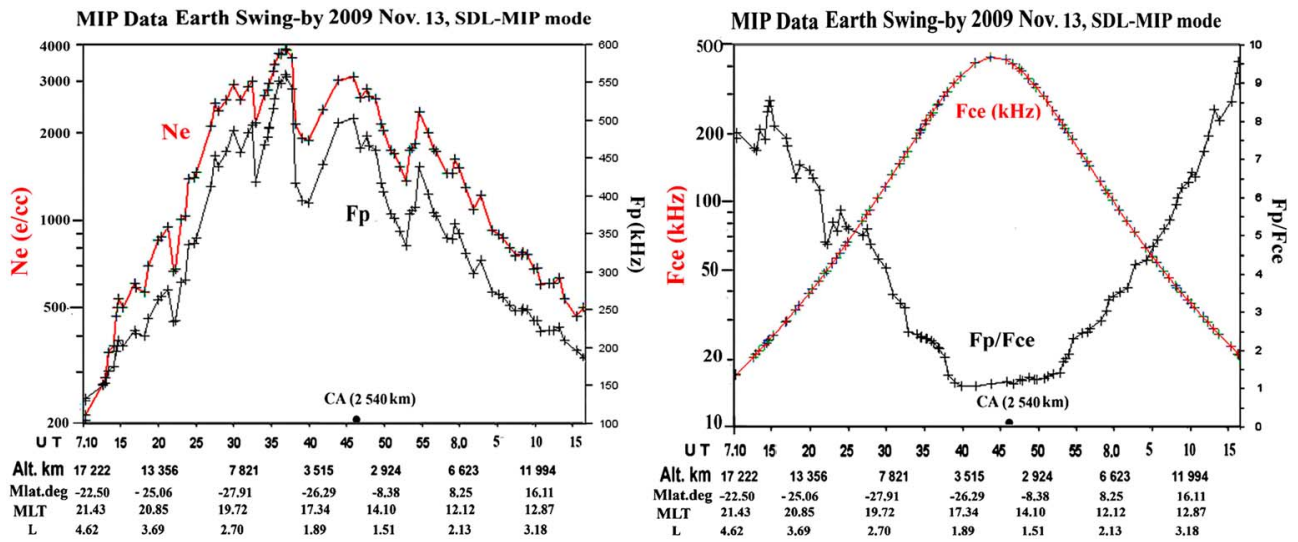

Figure 4. (left) Variations of plasma frequency $\left(F_{\mathrm{p}}\right)$ and electron density $\left(N_{\mathrm{e}}\right)$; (right) electron cyclotron frequency $\left(F_{\mathrm{ce}}\right)$ and $F_{\mathrm{p}} / F_{\text {ce }}$ ration. 


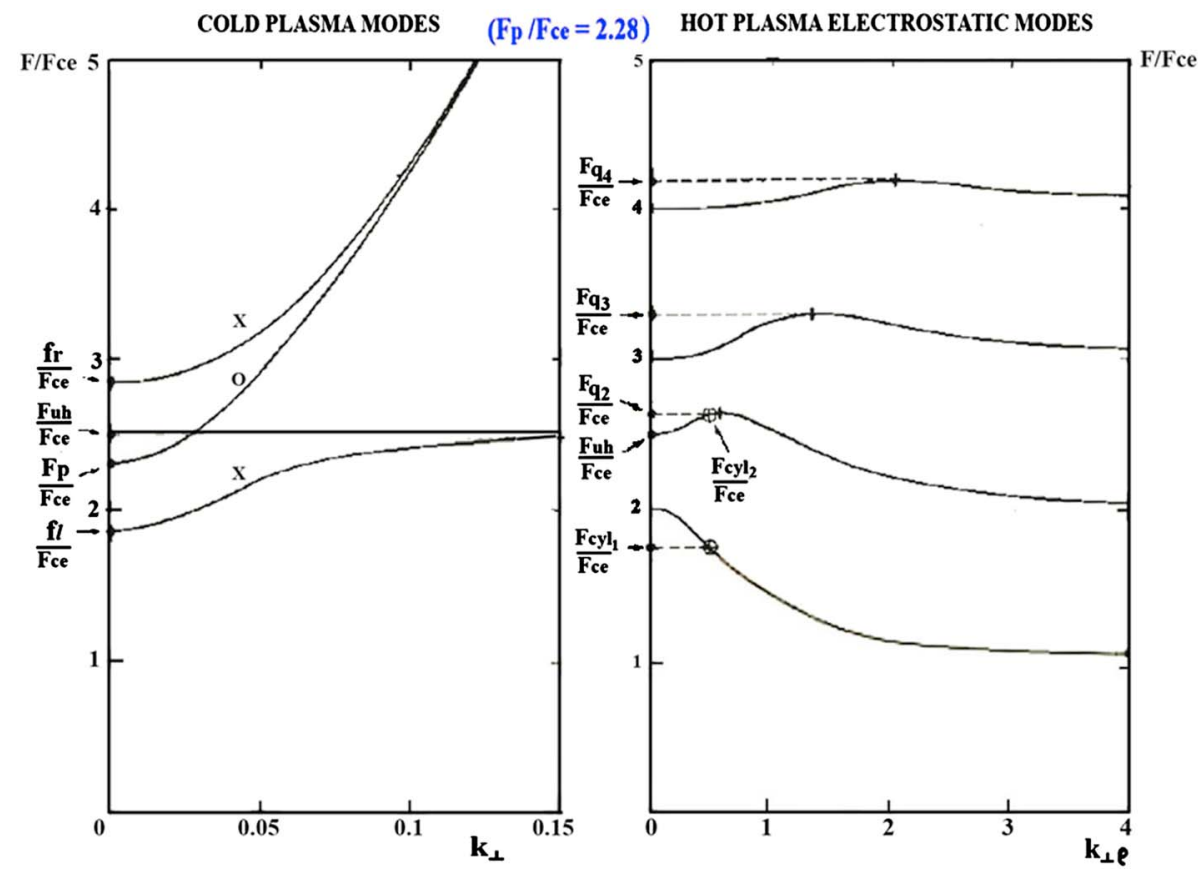

Figure 5. Dispersion curves from linear theory calculation. (left) Cold plasma electromagnetic modes: one ordinary (0), and two right and left polarized extraordinary modes (X). (right) Electrostatic Bernstein [1958] modes in a warm plasma.

\subsection{Summary Presentation of Fq's Bernstein Waves Observed With SDL-MIP Modes}

The electron Bernstein waves [Bernstein, 1958] propagating in magnetized thermal plasmas are electrostatic waves coupled with the singularities $\left(\mathbf{k}_{\perp} \rightarrow 0\right)$ of the wave-vector components perpendicular to $\mathbf{B}_{\mathbf{0}}$ of both extraordinary right- and left-polarized electromagnetic modes that are present in the magnetized cold plasmas (Figure 5, left). While the frequency dispersion law of each harmonic branch of the electrostatic waves (Figure 5 , right) is reaching an inflection point $\left(\mathrm{d} f / \mathrm{dk}_{\perp}=0\right)$, the wave group velocity vanishes, that characterizes the Fq resonance associated to the $n$th harmonic of $F_{\text {ce }}$ with $n<q<n+1$. Pioneering observations of $\mathrm{Fq}$ resonances triggered by ionospheric topside sounder experiments were performed on board the Alouette-2 satellite, at medium latitude and about $2200 \mathrm{~km}$ altitude [Muldrew, 1972]. For $F_{\mathrm{p}} / F_{\mathrm{ce}} \sim 2.2$, the wavelengths of $\mathrm{Fq} 2$ and $\mathrm{Fq} 3$ resonances at 0.95 and $1.3 \mathrm{MHz}$ were estimated about $1 \mathrm{~m}$ and $45 \mathrm{~cm}$, respectively (assuming electron temperature $T_{e}=3000^{\circ} \mathrm{K}$ ). Notice that such wavelengths could be about 50 and 100 smaller than the length of each of both wire antennas (22.8 and $45.5 \mathrm{~m}$ ) of the Alouette spacecraft.

Other methods have been proposed independently to estimate the electron temperature from the topside plasma resonances [e.g., Warnock et al., 1970; Feldstein and Graff, 1972], leading subsequently to a rough evaluation of the Larmor radius and the Debye length. However, the general feeling is actually that the quality factor of resonances triggered and received by using either active or passive experiments is closely depending on how much the wavelength is matching the size of the reception antenna [e.g., Perraut et al., 1990, and references therein].

The SDL preprogrammed operations of the 2009 Earth's swing by included successive sequential passive and active modes, allowing to highlight the differences between the characteristics of the natural emissions and that of the resonances triggered by the MIP active mode. The frequency spectrograms of natural waves observed during the SDL passive mode sequences (Figure 6), reveals, besides the three well-known harmonic of instrumental interference $(130,260$, and $520 \mathrm{kHz}$ ), the signatures of natural emissions that are the following:

1. likely extraordinary modes of first and second electron cyclotron harmonics (e.g., approximately at $7 \mathrm{~h}$ 33/38);

2. $F_{\text {cyl }}(1$ and 2$)$ and $F q(2,3, \ldots$ up to 9$)$;

3. VLF whistler mode from $7 \mathrm{kHz}$ to $F_{\text {ce. }}$ 


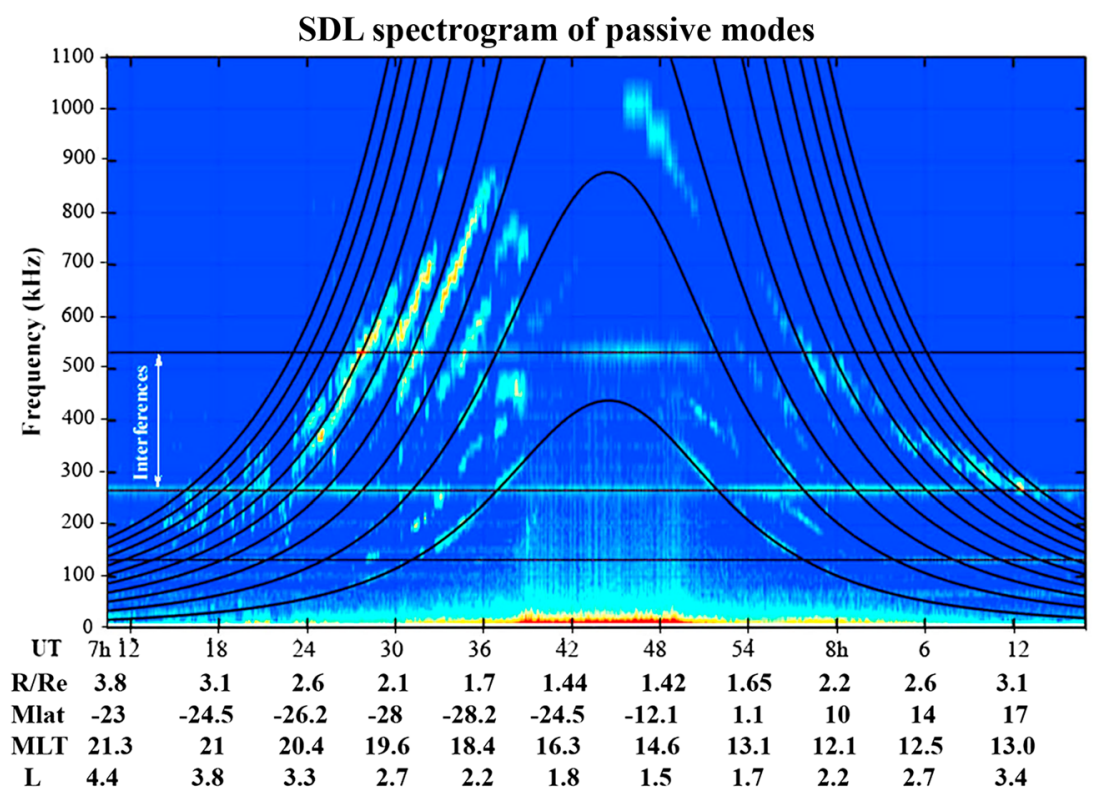

Figure 6. Smoothed black lines: $F_{\text {ce }}$ harmonics derived from RPC magnetometer. Pale blue to yellow: $F_{\text {cyl }}, \mathrm{Fq} 2$ to Fq9. Pale blue to red: VLF emission below $F_{\text {ce }}$. Black straight lines: instrumental interferences.

In the example presented in section 2.1 (Figure 3), the two Fq5 and Fq6 resonances are seen substantially at the same frequencies on both panels, taking into account the $30 \mathrm{~s}$ gap between both modes which are using different data sampling rates. However, the passive mode, contrary to the active one, does not exhibit any visible signature of the $F_{\mathrm{p}}$ and/or $F_{\mathrm{uh}}$ cold plasma resonances. This fact agrees with what is expected from the absence of stimuli. Another significant difference between both modes is the presence of a very low frequency (VLF) emission below $F_{\text {ce, }}$ visible on Figure 3 (left). The latter emission is thought indeed being associated with the presence of an electron temperature anisotropy, a fact that concerns the main topic treated in section 4.

\section{Determination of Fq's Parameters in a Maxwell-Boltzmann Plasma}

The first order approximation of the dispersion law for Fq's electrostatic waves in a magnetized isotropic thermal plasma, shown in Figure 5 (right), was derived by Pottelette et al. [1981], considering a kinetic Maxwellian distribution [e.g., Alexandrov et al., 1984]. In such conditions, the basic dispersion equation [Stix, 1962] reads

$$
\left.\delta\left(k_{/ /}, k_{\perp}\right)=\left(k_{/ /}^{2}+k_{\perp}^{2}\right) \rho^{2}+\left(\frac{F_{\mathrm{p}}}{F_{\mathrm{ce}}}\right)^{2} \sum_{m=-\mu}^{m=+\mu} I_{m}\left(k_{\perp}^{2} \rho^{2}\right) \exp \left(-k_{\perp}^{2} \rho^{2}\right)\left[1+\alpha_{0} f\left(\alpha_{m}\right)\right]\right)=0
$$

where $k_{/ /}$and $k_{\perp}$ are the parallel and perpendicular wave vector components, respectively, with respect to the steady magnetic field $B_{0} ; I_{m}$ is the modified Bessel function of first species and order $m$, and $\rho$ is the Larmor radius given by

$$
\rho=\left(m_{\mathrm{e}} K_{\mathrm{B}} T_{\mathrm{e} \perp}\right)^{1 / 2} / q_{\mathrm{e}} B_{0}
$$

in which $m_{e}$ and $q_{e}$ are the electron mass and electrical charge, respectively, $K_{\mathrm{B}}$ the Boltzmann constant, $T_{e \perp}$ the electron temperature component perpendicular to $B_{0}$, and $\varphi$ is the Fried and Conte [1961] plasma dispersion function with the argument $\alpha_{m}=\left[\left(f / F_{\mathrm{ce}}\right)-m\right] /\left(k_{/ /} \rho \sqrt{ } 2\right)$.

The solutions of the dispersion equation (equation (4)) that are considered in Figure 7 (right) and throughout this article were derived by Hamelin [1980] and Pottelette et al. [1981], and the data file for Fa $\mathrm{F}_{n}$ parameters from $n=2$ to 19 is available in the supporting information S1.

Here we are essentially concerned with the Fq's electrostatic modes (Figure 5, right), which imply the perpendicular components $k_{\perp}, T_{e \perp}$, and the Larmor radius $\rho$ (equations (4) and (5)). Since the first right-hand term of 
Earth Fly-By MIP-SDL Mode 2009.1I.13; 7 h. 24 min. $50.8 \mathrm{~s}$.

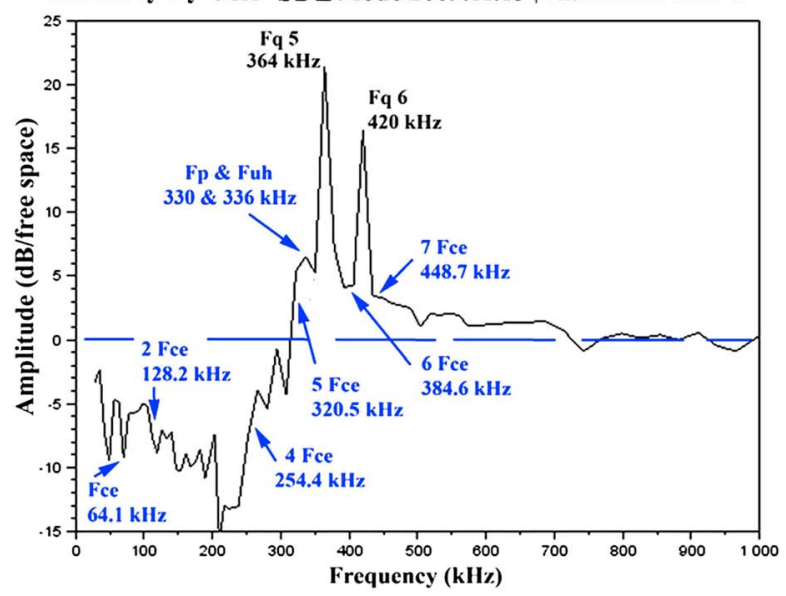

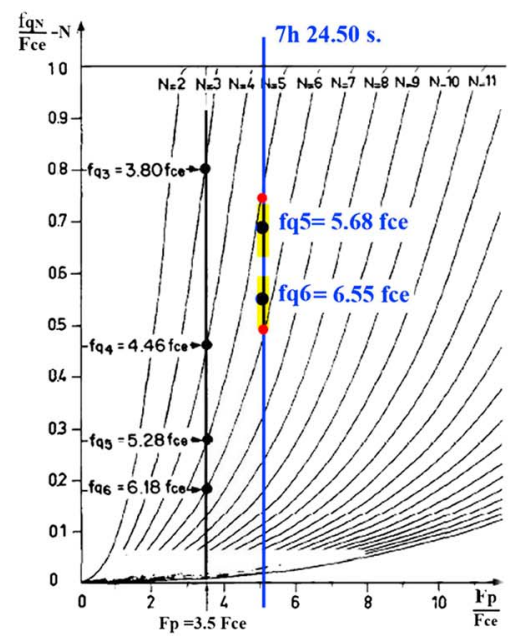

Figure 7. Checking Fq's responses compatibility with a Maxwellian plasma. (left) Active SDL mode exhibiting the same couples Fq5 and Fq6 as in Figure 3. (right) Checking measurements versus Maxwellian plasma distribution (see text).

the dispersion equation contains both $k_{\perp}$ and $k_{/ /}$components, we consider it being applicable to anisotropic plasmas as well, at least as a first approximation. However, we need to check how far the observed Fq's responses are compatible with a Maxwellian distribution, although we consider below a bi-Maxwellian distribution in terms of anisotropy.

First of all, we must account for the frequency resolution uncertainties which are essentially due to the synthesizer quantization size with 92 frequency steps ranging from 28 to $1582 \mathrm{kHz}$. Moreover, in the active mode the frequency is jumping successively by steps of 7, 14, 28, and $56 \mathrm{kHz}$ bins resolution and 75 steps from 7 to $1512 \mathrm{kHz}$ in the passive mode. It appears nevertheless that most of the 2009 flyby SDL data are found compatible indeed with a Maxwellian distribution as shown in Figure 7. For a Maxwellian distribution one should read Fq5 $=5.75 F_{\text {ce }}$ in Figure 7 (right), and Fq6 $=6.5 F_{\text {ce }}$ (red dots), i.e., $368.6 \mathrm{kHz}$ instead of 364, and $416.6 \mathrm{kHz}$ instead of 420 , respectively). Such differences are both consistent with $\pm 4 \mathrm{kHz}$ shift, which is even smaller than the $14 \mathrm{kHz}$ bandwidth of the synthesizer bins. Thus, both Fq's frequencies are marginally compatible with a Maxwellian distribution.

Moreover, the aim of this work is to get a confident estimate of the evolution of the electron temperature perpendicular component $T_{\mathrm{e} \perp}$, which involves the treatment of successive series of Fq's spectra from the SDL responses. We assume first that $T_{e_{\perp}}$ is keeping a fixed value during the sampling time duration of each SDL spectrum (less than $4 \mathrm{~s}$ ) exhibiting a couple of consecutive Fqs (e.g., Figure 7). Moreover, as long as the magnetic field amplitude also is assumed to not having changed significantly during such a short time, we assign to that couple obviously the same value of Larmor radius $\rho$ (equation (5)) for both Fqs. Since the values of $k_{\perp} \rho$ associated to each $\mathrm{Fq}_{n}$ are increasing with the order $n$ (Figure 5 , right), the size of wave vector $k_{\perp}$ must have grown accordingly. Therefore, in order to derive a common value of $\rho$ and subsequently $T_{e_{\perp}}$ for any SDL spectrum, one evaluates the average $k_{\perp} \rho$ contribution associated to each couple $\mathrm{Fq}_{n}$ and $\mathrm{Fq}_{n+1}$. Subsequently, we derive an average effective wavelength $\lambda_{n, n+1}$, which is supposedly integrating the timefrequency dispersion of both resonances, such as that seen in Figure 7 (right) for $\mathrm{Fq}_{5}$ and $\mathrm{Fq}_{6}$. Moreover, as we will see below (section 3.1 and Figure 9, right), for few transitions between two consecutive SDL spectra, one observes sudden $k_{\perp} \rho$ shifts interpreted as resulting from Rosetta spacecraft crossing successive series of FACs.

\subsection{Effective $k_{\perp} \rho$ Values and Associated Wavelengths of the $\mathrm{Fq}_{n} / \mathrm{Fq}_{n+1}$ Couples.}

From the solutions of the dispersion equation (equation (4)) applied to the three first Fq's resonances versus the dimensionless parameters $F_{\mathrm{p}} / F_{\mathrm{ce}}, \mathrm{Fq}_{n} / F_{\mathrm{ce}}-n$ and $k_{\perp} \rho$ plotted in Figure 8, one observes for any value of $F_{\mathrm{p}} /$ $F_{\text {ce }}$ that the successive values of $k_{\perp} \rho$, associated to the growing $n$ order, are increasing less and less rapidly. Such a behavior implies that the corresponding Fq's wavelength $\left(\lambda_{n} \equiv 2 \pi / k_{\perp}\right)$ should have to slightly decrease, while both $T_{e \perp}$ and $\rho$ are assumed to remain constant during the SDL sample. The latter behavior appears to 
Theoretical three-dimensional diagram of Fq's

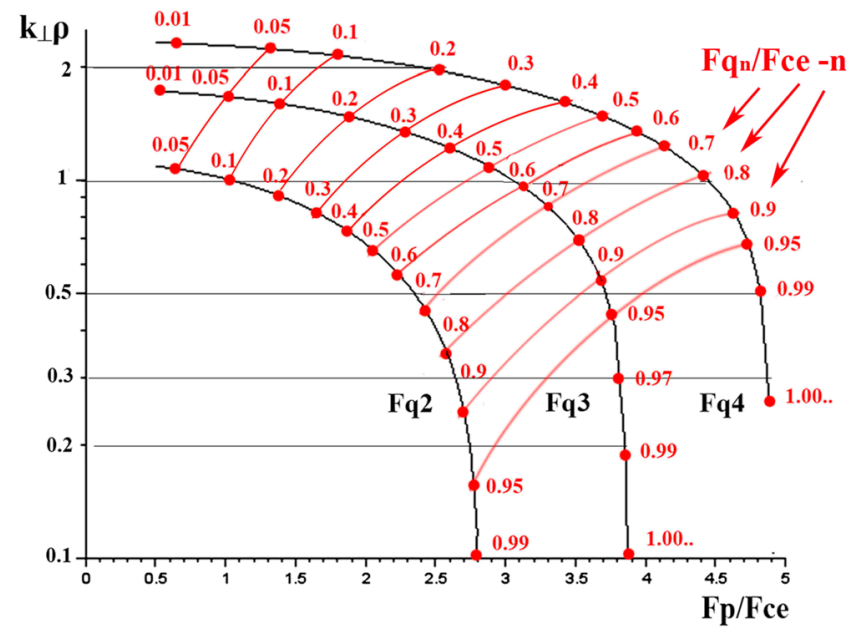

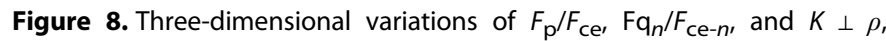
derived from the dispersion equation for $\mathrm{Fq} 2, \mathrm{Fq} 3$, and $\mathrm{Fq} 4$, in a Maxwellian plasma. account for the presence of one couple only for two consecutive Fqs in each SDL active mode spectrum. This behavior will be interpreted in section 3.2 as a self-consistent selection mechanism of the electrostatic wavelength by the length of the reception dipole.

Let us consider, for instance, the couple Fq5 and Fq6 seen in Figure 7. We define the common effective value $<k_{\perp} \rho>$ as

$$
\begin{aligned}
<k_{\perp} \rho> & =\rho \frac{k_{5}+k_{6}}{2} \\
& =2 \pi \rho \frac{\lambda_{5}+\lambda_{6}}{2 \lambda_{5} \lambda_{6}}=\frac{2 \pi \rho}{\lambda_{5 \approx 6}}
\end{aligned}
$$

where $\lambda_{5 \approx 6}$ is the effective electrostatic wavelength of the couple Fq5 and Fq6, defined as

$$
\lambda_{5 \approx 6}=\frac{2 \lambda_{5} \lambda_{6}}{\lambda_{5}+\lambda_{6}}
$$

then, the generalized expression applicable to all SDL responses of 2009 swing by reads as follows:

$$
\lambda_{n \approx n+1}=\frac{2 \lambda_{n} \lambda_{n+1}}{\lambda_{n}+\lambda_{n+1}}
$$

\subsection{Derivation of the Fq's Effective Wavelengths Excited by the SDL Active Mode}

The Fq's electrostatic cylindrical wavelengths are excited in the plane perpendicular to the local magnetic field and simultaneously induced along the $1 \mathrm{~m}$ long reception dipole (Figure 2). Then, the induced voltage depends upon the orientation of the dipole antenna with respect to the electrostatic wave field component perpendicular to $B_{0}$. So, the best fit occurs for the component which is also parallel to the antenna. Since we are benefiting from real-time data of the three components of magnetic field measured on board by the RPC fluxgate magnetometer [Glassmeier et al., 2007b], we get all necessary inputs in order to derive the properties of SDL response of Fq's resonances. The angles of the SDL dipole (Figure 2) versus the Rosetta spacecraft coordinates are

Parameters observed or inferred from Rosetta observations
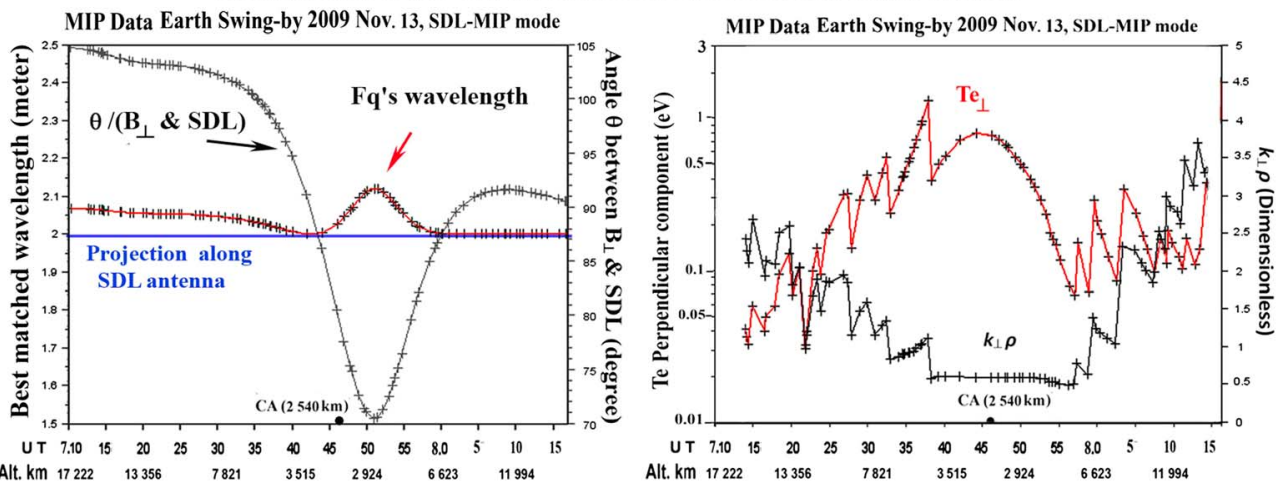

Figure 9. (left) Best matched wavelengths of Fq's couples and $\theta$ angle between $B_{0} \perp$ and SDL antenna. (right) Time profiles of $k \perp \rho$ and perpendicular electron temperature component $T_{\mathrm{e}} \perp$. 


$$
(\alpha ; x) \approx 90^{\circ} ;(\beta ; y) \approx 45^{\circ} ;(\gamma ; z) \approx 45^{\circ} .
$$

So, the SDL dipole antenna lies within the $y, z$ plane, and the angle $\theta$ between the reception dipole and the magnetic field vector $\boldsymbol{B}_{\mathbf{o}}$ (Figure 9, left) reads as follows:

$$
\cos \theta=\frac{B_{y} \cos 45^{\circ}+B_{z} \cos 45^{\circ}}{\left(B_{x}^{2}+B_{y}{ }^{2}+B_{z}^{2}\right)^{1 / 2}}
$$

where SC coordinates are the same for the RPC-MAG fluxgate magnetometer and the SDL probe.

We apply to the SDL receiving double-probe antenna the optimal transfer function observed with high-input impedance dipoles, i.e., the differential floating $A C$ voltages induced on both receiving electrodes are seen with opposite phases, according to the well-known node-antinode rule. Thus, the best fitted antenna sizes are odd multiples of one-half wavelength, as

$$
\lambda_{\mathrm{a}}=2 L /(2 \eta+1)
$$

where $L$ is the dipole length, $\lambda_{\mathrm{a}}$ is the wavelength component aligned with the SDL antenna, and $\eta$ is either 0 or an integer. Thus, with $L=1 \mathrm{~m}$ (Figure 2 ) the largest matched wavelength component fitting the antenna length is $\lambda_{\mathrm{a}}=2 \mathrm{~m}$, and the smallest wavelengths would be $0.66,0.4 \mathrm{~m}$, and so on. However, according to equations (5) and (6), such small sizes would lead to unrealistic values of electron temperature. For instance, at CA, where $k_{\perp} \rho=0.5$ (Figure 9, right), $\rho$ would be $\sim 5 \mathrm{~cm}$ and $T_{e_{\perp}}$ smaller than $5 \times 10^{-2} \mathrm{eV}$ instead of $0.7 \mathrm{eV}$ (Figure 9), and $T_{e / /}$ would be about 5 times smaller (equation (16)). Thus, we consider the above value $\left(\lambda_{\mathrm{a}}=2 \mathrm{~m}\right.$ ) being the most likely one fitting the effective electrostatic wavelength $\lambda_{n} \approx n+1$ of any Fq's couple (equation (8)). Knowing the variations of $\theta$ (equation (10)) during the SDL sequence plotted in Figure 9 (left), the largest best fitted Fq's wavelength component $\lambda_{\perp}$ perpendicular to $B_{0}$ is given by

$$
\lambda_{\perp}=2 / \sin \theta .
$$

\section{Derivation of Perpendicular Electron Temperature and $T_{e_{\perp}} / T_{e / /}$ Anisotropy}

Applying equation (6) to each Fq's couple of the entire SDL sequence, we obtain the variation profile of $k_{\perp} \rho$ (Figure 9, right), using the following generalized expression:

$$
<k_{\perp} \rho>=(1 / 2)\left[\left(k_{\perp} \rho\right]_{q n}+\left[\left(k_{\perp} \rho\right]_{q n+1} .\right.\right.
$$

Then, from the identity $k_{\perp} \equiv 2 \pi / \lambda_{\perp}$, and the values of $\theta$ (equation (10)), we get the values of the Larmor radius defined by equation (5), from which one obtains the variations profile of $T_{e \perp}$ plotted in Figure 9 (right). If we had assumed that the electron temperature was isotropic, i.e., $T_{e / /}=T_{e \perp}$, then the Debye length would be directly derived from the Larmor radius, as

$$
\lambda_{\mathrm{D}}=\rho F_{\mathrm{ce}} / F_{\mathrm{p}}
$$

However, the presence of natural VLF emissions, bounded at frequencies $f<F_{\text {ce }}$ (Figure 3, left) and observed throughout the entire SDL sequence (Figure 6), is well known in the Earth's magnetosphere to be a signature of the presence of the whistler-mode electron temperature anisotropy instability, with $T_{e \perp} / T_{e / /}>1$ [Kennel and Petschek, 1966; Gary, 1993].

\subsection{The Kinetic Source of $T_{e \perp} / T_{e / /}$ Anisotropy and Associated Dynamic Parameters}

The pioneering spacecraft observations of electron and ion temperature anisotropy in the Earth's ionosphere and magnetosphere, before Dynamics Explorer satellite measurements, were involving only thermal populations less than $1 \mathrm{eV}$ [Demars and Schunk, 1987]. After increasing improvements of spacecraft investigations and theoretical studies, the plasma dynamic domain was enlarged toward suprathermal and energetic populations up to $100 \mathrm{eV}$. Then, theoretical and simulation works were performed, intending to better characterize 
the dynamic range of $T_{e}$ and to understand the involved processes, including the source mechanisms of electron and/or ion temperature anisotropies.

Here we consider the electrostatic electron anisotropy instability, one of three instabilities driven by $T_{e \perp /} T_{e / /}$ $>1$ described by Gary and Cairns [1999]. According to this reference, this instability is the dominant growing mode driven by this anisotropy in the limit of $\beta_{/ / e}$ tending to 0 , where $\beta_{/ / e}$ is the electron thermal/magnetic pressure ratio "beta."

A few recent space missions have revealed the existence of electron or ion temperature anisotropy and have prompted numerical simulations of probable generation mechanisms, as, for instance, in regions of the Earth's magnetosheath and magnetic reconnection [Le et al., 2010], as well as in the solar wind [Štverák et al., 2008]. In the magnetosheath, the electron temperature anisotropy, as derived from in situ measurements performed with the plasma analyzer Peace instruments on board the four Cluster spacecraft C1, C2, C3, and C4 [Masood and Schwartz, 2005], was proposed to have been triggered by the whistler instability [Gary et al., 2005], although no data were unfortunately reported by the Cluster team about the eventual presence of any whistler emission.

The electron anisotropy instability leads the electric field fluctuations of the VLF whistler mode to scatter the electrons in the direction perpendicular to $B_{0}$. While the oblique $\boldsymbol{k}$ vector orientation versus the magnetic field is approaching the perpendicular direction, the instability tends toward a maximum growth rate. However, in our case, contrary to the Fq's resonances (section 3.2), we do not have any way to estimate the orientation of the unstable wave vector with respect to the magnetic field. Nevertheless, referring to Gary and Cairns [1999], using a linear approximation of the Vlasov's dispersion equation, these authors considered an average threshold condition defined as

$$
\omega_{\mathrm{r} /}\left|\Omega_{\mathrm{e}}\right| \equiv \gamma / F_{\mathrm{ce}} \approx 0.6, \text { for } F_{\mathrm{p}} / F_{\mathrm{ce}} \gg 1
$$

where $\omega_{r}$ is the real part of the threshold instability, $\Omega_{\mathrm{e}}$ is the angular electron-cyclotron frequency, and $\gamma$ is the real frequency threshold bounded between the electron and ion cyclotron frequencies. Applying the above relationship to an SDL sample of the passive mode response plotted in Figure 3 (left) with $F_{\text {ce }} \sim 53 \mathrm{kHz}$, the instability frequency threshold would lie at $\gamma \sim 32 \mathrm{kHz}$, i.e., $8 \mathrm{~dB}$ above the SDL free-space response reference, and $20 \mathrm{~dB}$ smaller than the lowest bound at $7 \mathrm{kHz}$. Moreover, the latter frequency is at least 4 times higher than the ion-cyclotron frequency cutoff. Thus, it appears that "the protons are not resonant and the mode properties are independent of $T_{e} / T_{p}$ and determined only by the two parameters $F_{\mathrm{p}} / F_{\mathrm{ce}}$ and $T_{e \perp} / T_{e / /}$ " [Gary and Cairns, 1999]. Then, the threshold condition for the electrostatic electron anisotropy instability in the limit of $\beta_{/ / e}$ tending to 0 represents an upper bound on the electron temperature anisotropy and can be written in the form of

$$
T_{\mathrm{e} \perp} / T_{\mathrm{e} / /}-1=2.95+\frac{0.25}{\left(F_{\mathrm{p}} / F_{\mathrm{ce}}-0.5\right)^{1.75}} .
$$

Introducing in equation (16) the variations of $F_{\mathrm{p}} / F_{\mathrm{ce}}$ throughout the entire SDL mode sequence (Figure 4, right), we get the anisotropy ratio $T_{\mathrm{e} \perp} / T_{\mathrm{e} / /}$ ranging from 3.95 to 4.6 (Figure 10, left). Then, knowing the values of $T_{\mathrm{e} \perp}$ (Figure 9), both profiles of $T_{\mathrm{e} \perp}$ and $T_{\mathrm{e} / /}$ are plotted together in Figure 10 (right). Although several theoretical modeling studies have been carried out (e.g., review for lonosphere-Plasmasphere; excluding aurora populations, by Demars and Schunk [1987]), no space experiment in the same regions confirmed such a thermal plasma anisotropy to date, to the authors knowledge.

Going furthermore for checking the self-consistency of our observations versus the Vlasov linear approximation model, we have introduced the electron temperature profiles in the equations of the dynamic parameters controlling the instability threshold, after Gary and Cairns [1999], namely, the nonrelativistic condition (also named electron temperature pressure):

$$
\alpha=\left(\frac{K_{\mathrm{B}} T_{\mathrm{e} / /}}{m_{\mathrm{e}} c^{2}}\right) \quad<<1,
$$

and the electron thermal/magnetic pressure ratio "beta" 

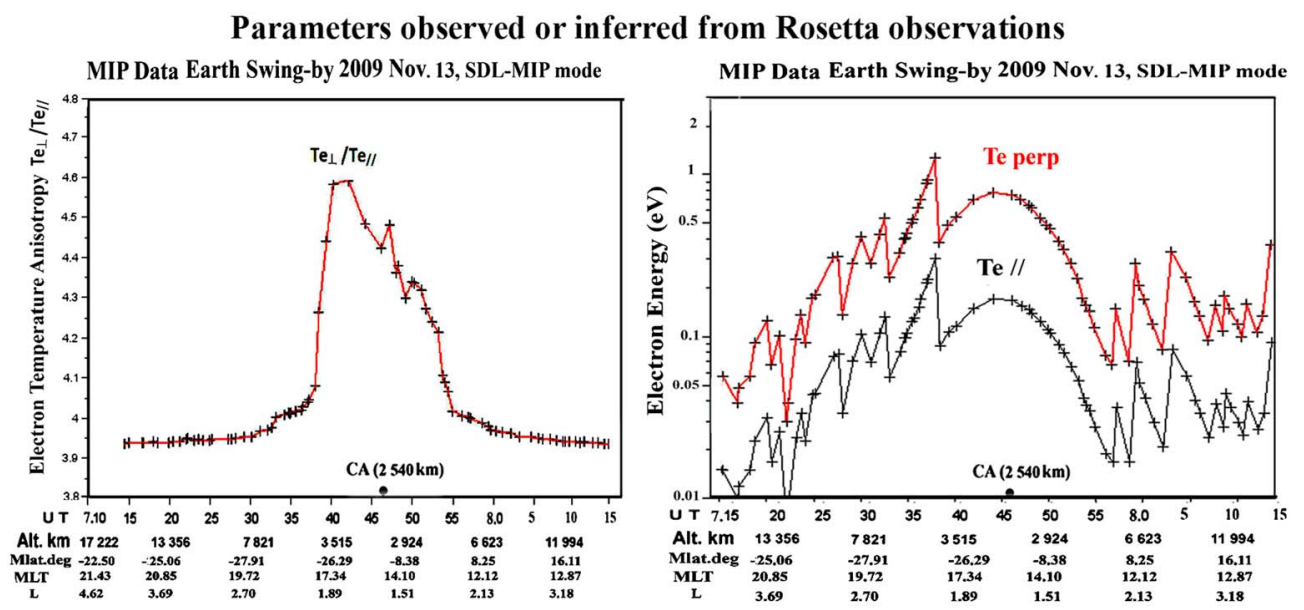

Figure 10. Electron temperature anisotropy deduced from linear theory [Gary and Cairns, 1999]. (left) Variations profile of $T_{\mathrm{e} \perp} / T_{\mathrm{e} / / ;}$ (right) Profiles of $T_{\mathrm{e} \perp}$ and $T_{\mathrm{e} / /}$ at the same scale.

$$
\beta_{/ / \mathrm{e}}=\left(\frac{2 K_{\mathrm{B}} T_{\mathrm{e} / /}}{m_{\mathrm{e}} c^{2}}\right)\left(\frac{F_{\mathrm{p}}}{F_{\mathrm{ce}}}\right)^{2} .
$$

The variation profiles of these two dynamic parameters plotted in Figure 11 compare satisfactorily with those seen on Figure 4a of the analytic model in Gary and Cairns [1999], within the same range of $T_{e \perp} / T_{e / /}$ ratio (from 3.95 to 4.6). By using the same method applied to solar wind data derived from the three different spacecraft: Helios 1, Cluster II, and Ulysses, Štverák et al. [2008] have derived a ratio $T_{e \perp} / T_{e / /}$ ranging between 0.2 and 2 , from 0.35 to $2 \mathrm{AU}$ (astronomical unit), confirming that the anisotropy energization processes in the solar wind are significantly different from those observed along the magnetospheric Rosetta trajectory.

We will consider now the possible implication of our results to the understanding of FACs tubes confinement mechanisms. First of all, it is worth noting the regular weak magnetic conditions occurring on 13 November 2009, with $K_{p}<1$ (NOAA base), excluding disturbed conditions such as magnetospheric substorms.

\subsection{FACs Structures and Confinement of Current Flow by Thermal Magnetic Pressure}

The large-amplitude sawtooth-shaped irregularities of $F_{\mathrm{p}}$ and $N_{\mathrm{e}}$ profiles (Figure 4, left), $T_{e \perp}$ and $T_{e / /}$ (Figure 10, right), $\alpha$ and $\beta_{/ / \mathrm{e}}$ (Figure 11), and their associated temporal gradients (Figure 12) are supporting our initial suggestion (Figure 1) that Rosetta was crossing a series of field-aligned currents. All consecutive couples with

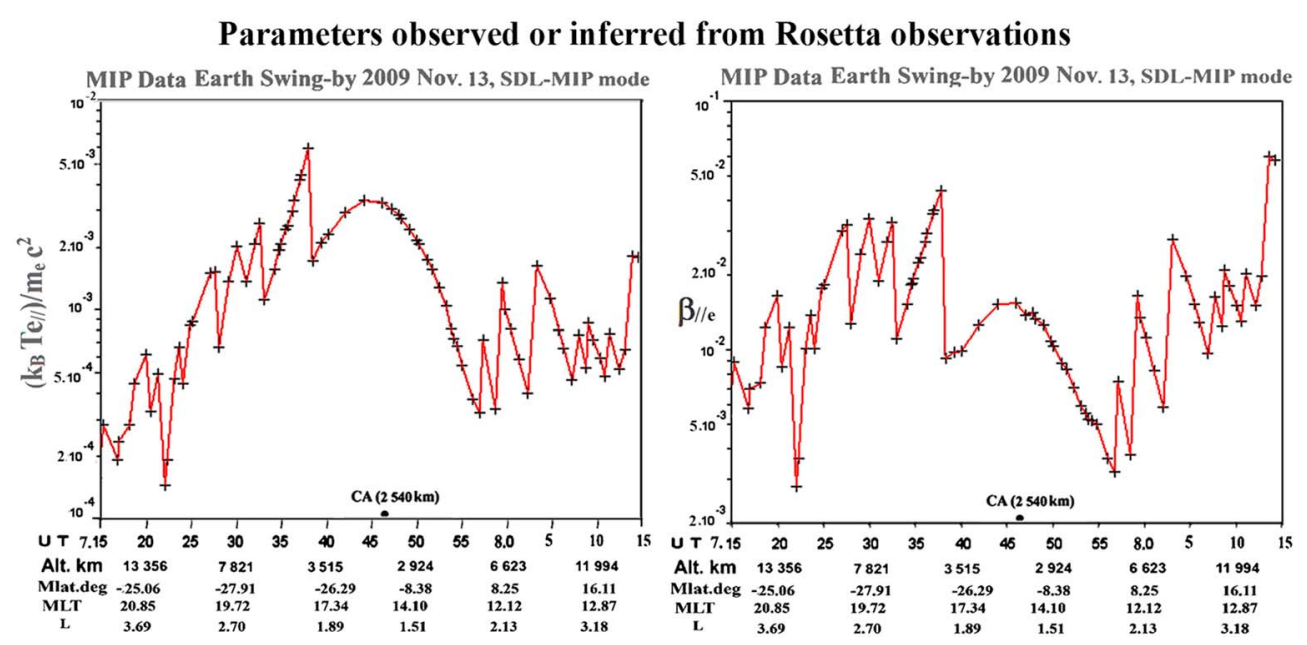

Figure 11. (left) Profile variations of the nonrelativistic condition $\left.\left(K_{\mathrm{B}} T_{\mathrm{e} / /}\right) / m_{\mathrm{e}} \mathrm{c}^{2} \ll 1\right)$. (right) Profile of $\beta / / \mathrm{e}$ (Parallel electron thermal magnetic pressure ratio "beta"). 
Parameters observed or inferred from Rosetta observations
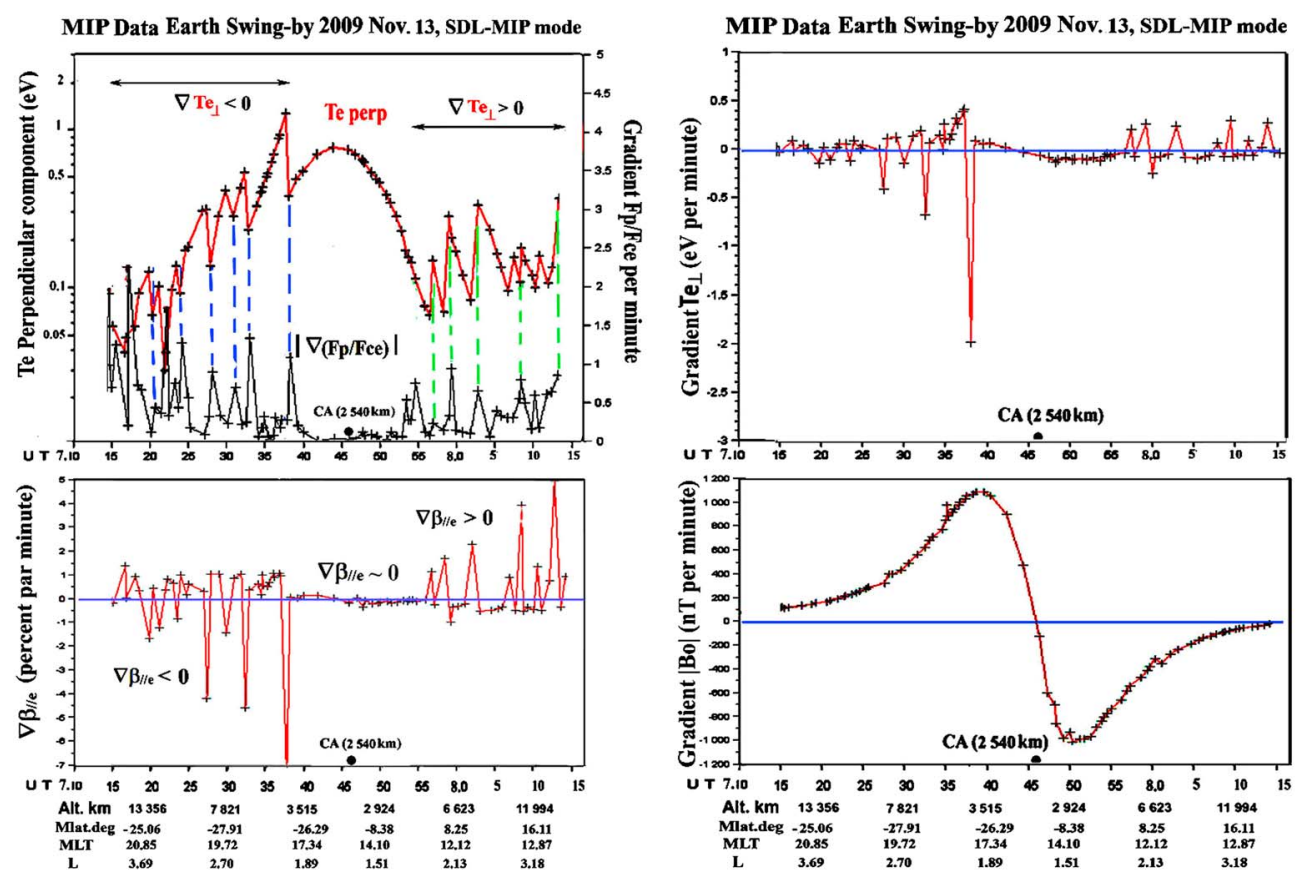

Figure 12. Temporal gradients associated to $F A C$ 's crossing. (top left) $T_{\mathrm{e} \perp}$ profile and $F_{\mathrm{p}} / F_{\mathrm{ce}}$ gradients; (bottom left) electron thermal/magnetic pressure $\beta_{/ / \mathrm{e}}$. (top right) $T_{\mathrm{e} \perp}$ gradient (eV/min); (bottom right) magnetic field gradient (nT/min).

highly transient gradients, such that those seen for instance at $7 \mathrm{~h} 33$ and $7 \mathrm{~h} \mathrm{38}$, are believed indeed being signatures of the spacecraft crossing sharp edges of individual FACs, with entry at $7 \mathrm{~h} 33$ and exit at $7 \mathrm{~h} 38$ in that case. At this point, we must note that it would be difficult to discriminate the FAC shape of a tube from that of a sheet, depending on whether the Rosetta trajectory was of a crossing or aligned type, so that the apparent FACs thickness should be considered as a maximum value. Nevertheless, both plots in Figure 1 are suggesting that most of cases are crossings.

Knowing the average cruise velocity of Rosetta at $7 \mathrm{~h} 35(\sim 12.5 \mathrm{~km} / \mathrm{s})$ and about $4000 \mathrm{~km}$ altitude, the particularly large structure observed at that time would be about $3750 \mathrm{~km}$ wide. Referring to the observations reported by Peria et al. [2000], performed on board the Fast Auroral Snapshot (FAST) spacecraft in the auroral acceleration region, although the Rosetta trajectory was different from that of FAST, the average extends of FACs, observed at about the same altitudes $(4000 \mathrm{~km})$, were both ranging within a similar scale standing from 1 to $5^{\circ}$ invariant magnetic latitude (ILAT) at about $75^{\circ}$ [Peria et al., 2000, Plate 1] and $\sim 30^{\circ}$ geomagnetic latitude for Rosetta.

Moreover, as one can check in Figure 12, all gradients of $F_{\mathrm{p}} / F_{\mathrm{ce},}, T_{e \perp}$, and $\beta_{/ / \mathrm{e}}$ exhibiting a significant amplitude are repeatedly negative before $7 \mathrm{~h} \mathrm{38}$, positive after $7 \mathrm{~h} 56$, and nearly zero or very weak in the vicinity of CA. Such behavior is consistent with the gradient profile of the magnetic field amplitude $\left|B_{0}\right|$ (Figure 12, bottom right), since this profile follows the curvature inversion of the field lines at around CA. Such behavior is consistent indeed with expected regular shape of the magnetic field dipolar model, including small deviations ranging from about 100 to $200 \mathrm{nT}$ associated to the most significant structures assigned to the presumed FACs. Although the magnitude of such deviations is again compatible with the 6 min sample duration of FAST data [Peria et al., 2000], it does not seem that so weak gradients of magnetic field amplitude could play a major role for controlling the squeeze of the large FACs structures. Instead, the ratio of pressure anisotropy $\beta_{\perp \mathrm{e}} / \beta_{/ / \mathrm{e}}>1$ appears as a better candidate to play that role because the gradient of $T_{e \perp}$ is suddenly dropping 1 order of magnitude through the supposed FAC structure (Figure 12, top right).

Referring to the contribution of Hall fields associated to an electron pressure anisotropy of the upstream current in the magnetic reconnection region [Le et al., 2010], the resulting $\boldsymbol{J} \times \boldsymbol{B}$ force is found lying perpendicular to both the current flow and the magnetic field tubes, and "The electron jets streaming near the thermal 
speed across the magnetic field are thus controlled by the electron pressure anisotropy," according to these authors. Conversely, with other space observations [e.g., Farrugia et al., 2016] in the situation of a transient reconnection flux transfer event (FTE], a magnetic pressure larger than the electron thermal is found accounting for a nonforce-free mechanism leading to squeeze the series of FACs. Notice that a similar behavior was observed during the Rosetta 2009 flyby, although deeper analysis of such mechanisms falls outside the purpose of this article.

Otherwise, another major question remains common to all spacecraft observations of FACs; that is, what is the amplitude of currents carried by the FAC tubes, and what could be the relative proportion between the thermal electrons seen by our instrument and the unmeasured high-energy particles distributions? Here again, a deeper analysis and numerical simulations about those questions fall outside the prime purpose of this paper and should have to be considered in the frame of other space missions in the same regions.

\section{Summary and Conclusion}

The Rosetta MIP instrument observations using the SDL mode in the Earth's magnetosphere have revealed series of demarcated field-aligned beam currents of thermal plasma. The latter structures exhibit all plasma properties of an electron temperature anisotropic distribution. Such results, obtained from a nonconventional operating mode of the mutual impedance technique, provide a new valuable support to theoretical and experimental knowledge of the thermodynamic processes of field-aligned currents of the Earth's magnetosphere [e.g., review by Y. Kamide, 1982].

Several dynamic features of the FACs tubes crossed by Rosetta have been revealed. Although predicted by simulation works, these structures have been rarely observed and described in the magnetosphere with such detailed properties and size resolution, to the authors knowledge. Those features involve, in particular, spatiotemporal gradients of the thermal electron density, of the electron temperature anisotropy components, and of the electron thermal and magnetic pressure components associated to the FAC tubes or sheets structures. The physical returns of our findings, in terms of thermodynamic exchange and coupling mechanisms between successive FACs, were briefly analyzed in the frame of theoretical models and other space observations. Our most significant results were derived from an original application of the Fq's resonances properties. These results bring a significant contribution to the knowledge of the anisotropic electron thermal population components of the field-aligned currents in the magnetospheric regions that were crossed par Rosetta during geophysical quiet conditions and should give rise to future investigations.

As a brief conclusion, the new approach proposed in this work for the treatment of Fq's resonances appears promising to be implemented for further space missions in magnetized plasmas of the planets in the solar system, in order to determine the electron temperature perpendicular component, to reveal the possible existence of an electron temperature anisotropy, and thus the force ratio between the two components of electron and magnetic thermal pressures.

Acknowledgments

The authors are grateful to the RPC-Mag team for the precious fluxgate magnetometer data used in this study. Many thanks from Christian Béghin to Donald Muldrew for his expert advice about Alouette satellite measurements and valuable discussions. Please contact Karl-Heinz Glassmeier with questions about the flux-gate magnetometer archive data at kh.glassmeier@tu-bs.de.

\section{References}

Alexandrov, A. F., L. S. Bogdankevich, and A. A. Rukhadze (1984), Principles of Plasma Electrodynamics, 488 pp., Springer New York. Béghin, C. (1971), Excitation de la Résonance Hybride Basse (LHR) par sonde quadripolaire à bord d'une fusée, pp. 1071-1078, Space Research $\mathrm{X} 1$, Academic Verlag.

Béghin, C., and R. Debrie (1972), Characteristics of the electric field far from and close to a radiating antenna around the lower hybrid resonance in the ionospheric plasma, J. Plasma Phys., 8(3), 287-310.

Béghin, C., et al. (1976), Electron Temperature and Density Profiles, and Fluctuations of Electron Density Obtained by a Rocket Experiment in the Polar lonosphere, pp. 87-90, Space Research, XV1, Academic, Berlin.

Béghin, C., J. F. Karczewski, B. Poirier R. Debrie, and N Massevitch (1982), The Arcad-3 ISOPROBE experiment for high time resolution thermal plasma measurements, Ann. Geophys. 58(5), 615-629.

Bernstein, I. B. (1958), Waves in a plasma in a magnetic field, Phys. Rev., 109, 10.

Blanc, M., R. Kallenbach, and N. V. Erkaev (2005), Solar System magnetospheres, Space Sci. Rev., 116, $227-298$.

Carr, C., et al. (2007), RPC: The Rosetta plasma consortium, Space Sci. Rev., 128(1), 629-647.

Chasseriaux, J. M., R. Debrie, and C. Renard (1972), Electron density and temperature measurements in the lower ionosphere as deduced from the warm plasma theory of the h.f. quadrupole probe, Aust. J. Plant Physiol., 8(3), 231-253.

Christiansen, P., P. Gough, G. Martelli, J. J. Bloch, N. Cornilleau J. Etcheto, R. Gendrin, D. Jones, C. Béghin and P. Décréau (1978), Geos 1: Identification of natural magnetospheric emissions, Nature, 272, 682-686. 
Décréau, P. M. E, C. Béghin and M. Parrot (1978), Electron density and temperature, as measured by the mutual impedance experiment on board Geos 1, Sci. Rev. 22, 581-595.

Demars, H. G., and R. W. Schunk (1987), Temperature anisotropies in the terrestrial ionosphere and plasmasphere, Rev. Geophys., 25(8), $1669-1679$

Dungey, J. W. (1961), Interplanetary magnetic field and the auroral zones, Phys. Rev. Lett., 6, 47.

Farrugia, C. J., et al. (2016), Magnetospheric Multiscale mission observations and non-force free modeling of a flux transfer event immersed in a super-Alfvénic flow, Geophys. Res. Lett., 43, 6070-6077, doi:10.1002/2016GL068758.

Feldstein, R., and P. Graff (1972), Daytime and nighttime electron temperatures from topside resonances, J. Geophys. Res., 77(10), 1896-1904. Fried, B. D., and S. D. Conte (1961), The Plasma Dispersion Function, Academic Press, New York.

Gary, S. P. (1993), Theory of Space Plasma Microinstabilities, Cambridge Univ. Press, New York.

Gary, S. P., and I. H. Cairns (1999), Electron temperature instabilities; whistler, electrostatic and Z mode. J. Geophys. Res., 104(A9), $19,835-19,842$

Gary, S. P., B. Lavraud, M. F. Thomsen, B. Lefebvre, and S. J. Schwartz (2005), Electron anisotropy constraint in the magnetosheath: Cluster observations, Geophys. Res. Lett., 32, L13109, doi:10.1029/2005GL023234.

Geiswiller, J., J. G. Trotignon, C. Béghin, and E. Kolesnikova (2001a), Rosetta mission mutual impedance probe modelling: The short and long Debye length plasma cases, Astrophys. Space Sci., 277, 317-318.

Geiswiller J., C. Béghin, E. Kolesnikova, D. Lagoutte, J. L. Michau, and J. L. Trotignon (2001b), Rosetta spacecraft influence on the mutual impedance probe frequency response in the long Debye length mode, Planet. Space Sci., 49, 633-644.

Glassmeier, K. H., H. Boehnhardt, D. Koschny, E. Kührt, and I. Richter (2007a), The Rosetta mission: Flying towards the origin of the solar System, Space Sci. Rev., 128, 1-21, doi: 10.1007/s11214-006-9140-8.

Glassmeier, K. H., et al. (2007b), RPC-MAG, The fluxgate magnetometer in the ROSETTA consortium, Space Sci. Rev., 128, 649-670.

Hamelin, M. (1980), Propagation and direction radiation properties of the cyclotron harmonic waves in a Maxwellian plasma, Communication at International Conference on Plasma Physics, Nagoya.

Kamide, Y. (1982), The relationship between field-aligned currents and the auroral electrojets: A review, Space Sci. Rev., 31, 127-243.

Kennel, C. F., and H. E. Petschek (1966), Limit on stably trapped particle fluxes, J. Geophys. Res., 71, 1.

Le, A., J. Egedal, W. Daughton, J. F. Drake, W. Fox, and N. Katz (2010), Magnitude of the Hall fields during magnetic reconnection, Geophys. Res. Lett., 37, LO3106, doi:10.1029/2009GL041941.

Lijima, T. , and T. A. Potemra (1976), The amplitude distribution of field-aligned currents at northern high latitudes observed by tried J. Geophys. Res., 81, 2165-2174.

Masood, W., and S. J. Schwartz (2005), Observations of the development of electron temperature anisotropies in the Earth's magnetosheath J. Geophys. Res. 113, A01216, doi:10.1029/2007JA012715.

Michel, E., C. Béghin, A. Gonfalone, and I. F. Ivanov (1975), Mesures de densité et de température électronique sur fusée dans l'ionosphère polaire par l'étude du cône de résonance, Ann. Geophys., 31(4), 463-471.

Milan, R. M., and R. M. Thorne (2007), Review of radiation belts relativistic electron losses, J. Atmos. Sol. Terr. Phys., 69(3), $362-377$.

Mozer, F. S. C. A. Cattell, R. B. Temerin, R. B. Tobert, S. Von Glinski, M. Woldorff and J. Wygant (1979), The dc and ac electric field, plasma density, plasma temperature, and field-aligned current experiments on the S3-3 satellite, J. Geophys. Res., 84(A10), 5875-5884, doi:10.1029/JA084iA10p05875.

Muldrew, D. B. (1972), Electrostatic resonances associated with the maximum frequencies of cyclotron-harmonic waves, J. Geophys. Res. 77(10), 1794-1801.

Peria, W. J., C. W. Carlson, and R. E. Ergun (2000), Characteristics of field-aligned currents near the auroral acceleration region: FAST observations, in Magnetospheric Current Systems, edited by S.-I. Ohtani, et al., pp. 1-20, AGU, Washington, D. C., doi:10.1029/GM118p0181.

Perraut, S., H. de Feraudy, A. Roux, M. E. Décreau, J. Paris, and L. Matson (1990), Density measurements in key regions of the Earth's magnetosphere, cusp and auroral regions, J. Geophys. Res., 95(A5), 5997-6014.

Pottelette, R., M. Hamelin, and J. M. Illiano (1981), Interpretation of the fine structure of electrostatic waves excited in space, Phys. Fluids, 24(8) $1517-1526$

Stix, T. H. (1962), The Theory of Plasma Waves, 283 pp., McGraw-Hill, New York.

Storey, L. R. O., M. P. Aubry, and P. Meyer (1969), A quadrupole probe for the study of ionospheric plasma resonances, in Plasma Waves in Space and in the Laboratory, vol. 1, pp. 303-332, Edinburg Univ. Press.

Štverák, Š., P. Trávniček, M. Maksimovic, E. Marsch, A. N. Fazakerley, and E. Scime (2008), Electron temperature anisotropy constraints in the solar wind, J. Geophys. Res., 113, A03103, doi:10.1029/2007JA012733.

Trotignon, J. G., et al. (2007a), RPC-MIP: The mutual impedance probe of the Rosetta plasma consortium, Space Sci. Rev., 128, 713-728.

Trotignon, J. G., X. Vallieres, D. Lagoutte, S. Grimald, J. P. Lebreton, and R. Patrick (2007b), The MIP experiment of the Rosetta orbiter: A mutual impedance probe for waves and plasma diagnosis. Advances in geosciences, Planet. Sci., 15, 323-341, AOGS.

Warnock, J. M., J. R. McAfee, and T. M Thompson (1970), Electron temperature from topside plasma resonance observations, J. Geophys. Res., 75(34), 7272-7275. 